\title{
Treating challenging behavior or aggression in people with intellectual disability: Are antipsychotics or antidepressants the preferred choice?
}

\author{
Beghi $\mathrm{M}^{1 *}$, Beghi $\mathrm{E}^{2}$, Cornaggia $\mathrm{CM}^{3}$, Perin $\mathrm{C}^{3}$ and Lassi $\mathrm{S}^{4}$ \\ ${ }^{1}$ Department of Mental Health, AUSL Romagna, Ravenna, Italy \\ ${ }^{2}$ Department of Neurosciences, Istituto di Ricerche Farmacologiche Mario Negri IRCSS; Milano, Italy \\ ${ }^{3}$ School of Medicine and Surgery, University of Milano Bicocca, Italy \\ ${ }^{4}$ Fondation Autisme Luxembourg, Luxembourg, Italy
}

Intellectual disability (ID) occurs in $1 \%$ of the general population [1]. The prevalence of challenging behavior in these patients is high (6.1\% in the community and $40 \%$ in long-stay hospital, [2]), as is the prevalence of aggression. (51.8\%), even if only $4.9 \%$ of individuals display aggressive behavior leading to injury [3]. A growing number of adults with ID are today reaching old age. In a survey conducted in patients with ID over 40 years of age, over half of the sample displayed any type of behavioral problems [4]. Aggression involves a variety of psychological processes and progress has been made in understanding the increasing brain mechanisms [5]. However, the neurotransmitters Serotonin, Dopamine and $\gamma$-Aminobutyric acid seem to have a role in aggression but so far predictions cannot be made with confidence about drug effects on aggression [5]. There is paucity of randomized trials for the treatment of persistent aggressive behavior. Antipsychotic drugs, particularly the atypical group, have been the most commonly used interventions in recent years, [6,7] even in absence of evidencebased data. In a UK survey, [8] 64\% of the total sample were prescribed antipsychotic medications, of whom almost half (49\%) had a schizophrenia spectrum or affective disorder diagnosis, while a further third (36\%) exhibited behaviors recognized by the National Institute for Health and Clinical Excellence (NICE) as potentially legitimate targets for such treatment such as violence, aggression or self-injury. In a recent survey, [9] 39.2\% of adults were dispensed an antipsychotic medication, which increased to $56.4 \%$ in a sub-cohort residing in group homes. Even in this case, almost one-third (28.9\%) of people been prescribed an antipsychotic medication did not have a documented psychiatric diagnosis. Very little evidence can be found for the interventions of anger and behavioral management; the data available on these interventions come primarily from studies conducted in children in whom the behavior is part of the autistic spectrum; [7] moreover a randomized trial showed no benefits for either Risperidone or Haloperidol compared with placebo, with even some evidence of a better response to placebo than either active drug in the reduction of aggression. [10]

Another common practice not supported by evidence is polypharmacy: in a prospective study, [11] a high proportion (45\%) received more than one (polypharmacy) psychotropic medication at baseline and $41 \%$ at 6 months. As for antipsychotics, a similar proportion received them at baseline (75\%) and 6 months (73\%), with polypharmacy remaining similar at baseline $(10 \%)$ and at 6 months (9\%). Although higher doses of antipsychotics were positively correlated with more severe aggressive behavior, physical aggression towards objects, self-injurious behavior and increasing age, there was no significant correlation between mean aggression severity score change and antipsychotic daily dose change between baseline and 6 months. Older people with ID were more likely than the general population to be prescribed medications with anticholinergic effects, [12] intermediateor long-acting Benzodiazepines, and antipsychotics especially at the presence of a comorbid cognitive impairment (antipsychotics $50 \%$ vs $39 \%$ without dementia and benzodiazepine derivatives $55 \%$ vs $36 \%$ respectively) [12]. By contrast, $18.1 \%$ of adolescents received pharmacotherapy with $11.8 \%$ receiving monopharmacy, $6.4 \%$ and $5.9 \%$ simultaneously receiving multiple classes of medications. Polypharmacy is a significant concern for adults (and, in particular, in older adults) with ID. In fact, patients with ID seem at higher risk of side effects compared to the general population. A recent survey done in UK primary care setting [13] found asignificantly greater number of movement disorders in people with ID compared with those without, with parkinsonism and akathisia showing the greatest difference and neuroleptic malignant syndrome being three times more common. Respectively, $13 \%$ of the patients without psychotropic drugs and $61 \%$ of the patients with 2 psychotropic drugs had adverse events. Adverse events had a significantly negative influence on quality of life. [14] Physical wellbeing was negatively associated with parkinsonism, urinary problems, dysphagia and temperature dysregulation possibly due to antipsychotics use [15]. Moreover, rates of sleep problems in adults with ID ranged from $8.5 \%$ to $34.1 \%$ [16]. A prevalence of $9.2 \%$ was reported for significant sleep problems. Sleep problems were associated with challenging behavior, respiratory disease, visual impairment, psychiatric conditions, and using psychotropic, antiepileptic and/or antidepressant medication [16].

A recent audit conducted in the Netherlands concluded that both psychiatrists and family physicians are willing to discontinue their prescriptions in $51 \%$ of cases, varying from $22 \%$ to $87 \%$ per service

*Correspondence to: Massimiliano Beghi, Center of Mental Health, AUSL Romagna, piazza della Magnolia 5, Ravenna, Italy, E-mail: massimilianobeghi@auslromagna.it

Key words: psychotropic drugs, mental retardation, treatment, behavioral problems

Received: August 12, 2019; Accepted: August 30, 2019; Published: September 03,2019 
provider [17]. The variables "a living situation with care and support" and "challenging behavior" were associated with a higher chance of discontinuation. The main reasons for decisions not to discontinue were concerns for symptoms of restlessness, the presence of an autism spectrum disorder, previously unsuccessful attempts to discontinue and objections against discontinuation by legal representatives. Data available for discontinuation are scarce but encouraging [15]. Physical well-being improves in people who discontinue antipsychotic medication. Social functioning and mental well-being decrease after incomplete discontinuation but recovers at follow-up [15].

For these reasons the guidelines of the NICE claim that antidepressants are the first-choice medicines in the treatment of behavioral disorders associated to ID (www.nice.org.). As far as antidepressants are concerned, a systematic review by Sophanal et al. [18] studied the results of one crossover randomized controlled trial in a small cohort, seven prospective uncontrolled trials and two retrospective studies. Of these, one explored the effectiveness of Clomipramine, and nine considered various selective serotonin reuptake inhibitors (Fluoxetine, Paroxetine and Fluvoxamine). Antidepressants showed improvement of aggression and self-injurious behavior on average in less than $50 \%$ of cases. The efficacywas most pronounced in the presence of an underlying anxiety or an associated diagnosis of obsessive-compulsive disorder. Most studies used antidepressants as add-on therapy to other psychotropics. Therefore, it was not possible to know of any confounding effects of the concomitant medication. The authors did not conclude that antidepressants are ineffective, but the evidence was of scarce quality to evaluate their usefulness. Since, then, the burden of literature on this topic remained poor and limited to specific syndromes. A recent study [19] evaluated the effects of antidepressants on longevity, age at dementia onset, and survival after onset among adults with Down syndrome. Proportional hazards models showed a significant delay of onset among those taking antidepressants. Mean age at death or at end of study for those receiving antidepressants was 54.71 years; among others, it was 52.60 years. In the 35 adults with late-onset seizures and dementia who died, mean survival after seizure onset was 4.23 years. A study done in 2012 [20] found that in people with Williams syndrome, $24 \%$ of the individuals had been prescribed an SSRI medication, while $12 \%$ received another type of antidepressant or anxiolytic. $81 \%$ of respondents indicated that SSRI medications were either "Helpful" or "Somewhat Helpful", with paroxetine reported to be the least helpful. Sixty-four percent (64\%) of survey participants reported that non-SSRI antidepressants and anxiolytics were either "Helpful" or "Somewhat Helpful" in treating symptoms of anxiety.

In conclusion, the efficacy of antidepressantscertainly deserves more attention in research, as there is evidence suggesting that they can be used to manage behavior problems in adults with ID. Randomized trials on antidepressants in this specific population are needed to confirm the efficacy of these drugs and avoid the current misuse of antipsychotics drugs. Based on present evidence, the use of psychotropic drug treatment should be spared and reserved to those patients who put themselves at particular risk as a consequence of their behavior; such treatment should be regarded as temporary and as adjunctive to other forms of management. Discontinuation is possible and an improvement could be seen in the long term, even despite a worsening of behavior immediately after discontinuation.

It's clear that all other principal indications for psychotropic medications prescription, remain valid, paying special attention to difficulties in assessing psychiatric disorders in people with intellectual disability [21].

\section{References}

1. American Psychiatric Association. Diagnostic and Statistical Manual of Mental Disorders (DSM-5). 5th ed. Washington (DC): American Psychiatric Press; 2013.

2. Ali A, Blickwedel J, Hassiotis A (2014) Interventions for challenging behaviour in intellectual disability. Advances in psychiatric treatment 20: 184-192.

3. Crocker AG, Mercier C, Lachapelle Y, Brunet A, et al. (2006) Prevalence and types of aggressive behaviour among adults with intellectual disabilities. $J$ Intellect Disabil Res 50: 652-661.

4. O'Dwyer C, McCallion P, Burke É, Carroll R, et al. (2018) Prevalence and associated factors of problem behaviours among older adults with intellectual disabilities in Ireland. Res Dev Disabil 80: 192-204.

5. Willner $P$ (2015) The neurobiology of aggression: implications for the pharmacotherapy of aggressive challenging behaviour by people with intellectual disabilities. $J$ Intellect Disabil Res 59: 82-92.

6. Ji NY, Findling RL (2016) Pharmacotherapy for mental health problems in people with intellectual disability. Curr Opin Psychiatry 29: 103-125.

7. Oliver-Africano P, Murphy D, Tyrer P (2009) Aggressive behaviour in adults with intellectual disability: defining the role of drug treatment. CNS Drugs 23: 903-913.

8. Paton C, Bhatti S, Purandare K, Roy A, et al. (2016) Quality of prescribing of antipsychotic medication for people with intellectual disability under the care of UK mental health services: a cross-sectional audit of clinical practice. BMJ Open 6: e013116

9. Lunsky Y, Khuu W, Tadrous M, Vigod S, et al. (2018) Antipsychotic Use With and Without Comorbid Psychiatric Diagnosis Among Adults with Intellectual and Developmental Disabilities. Can J Psychiatry 63: 361-369.

10. Tyrer P, Oliver-Africano PC, Ahmed Z, Bouras N, et al. (2008) Risperidone, haloperidol, and placebo in the treatment of aggressive challenging behaviour in patients with intellectual disability: a randomised controlled trial. Lancet 371: 57-63.

11. Deb S, Unwin G, Deb T (2015) Characteristics and the trajectory of psychotropic medication use in general and antipsychotics in particular among adults with an intellectual disability who exhibit aggressive behaviour. J Intellect Disabil Res 59: 11-25.

12. Axmon A, Sandberg M, Ahlström G, Midlöv P (2017). Prescription of potentially inappropriate medications among older people with intellectual disability: a register study. BMC Pharmacol Toxicol 18: 68

13. Sheehan R, Horsfall L, Strydom A, Osborn D, et al. (2017) Movement side effects of antipsychotic drugs in adults with and without intellectual disability: UK populationbased cohort study. BMJ Open 7: e017406.

14. Scheifes A, Walraven S, Stolker JJ, Nijman HL, et al. (2016) Adverse events and the relation with quality of life in adults with intellectual disability and challenging behaviour using psychotropic drugs. Res Dev Disabil 49-50: 13-21.

15. Ramerman L, Hoekstra PJ, de Kuijper G (2018) Health-related quality of life in people with intellectual disability who use long-term antipsychotic drugs for challenging behaviour. Res Dev Disabil 75: 49-58.

16. van de Wouw E, Evenhuis HM, Echteld MA (2012) Prevalence, associated factors and treatment of sleep problems in adults with intellectual disability: a systematic review. Res Dev Disabil 33:1310-1332.

17. de Kuijper GM, Hoekstra PJ (2017) Physicians' reasons not to discontinue long-term used off-label antipsychotic drugs in people with intellectual disability. $J$ Intellect Disabil Res 61, 899-908.

18. Sohanpal SK, Deb S, Thomas C, Soni R, et al. (2007) The effectiveness of antidepressant medication in the management of behaviour problems in adults with intellectual disabilities: a systematic review. J Intellect Disabil Res 51: 750-765.

19. Tsiouris JA, Patti PJ, Flory MJ (2014) Effects of antidepressants on longevity and dementia onset among adults with Down syndrome: a retrospective study. J Clin Psychiatry 75: 731-737

20. Martens MA, Seyfer DL, Andridge RR, Foster JE, et al. (2012) Parent report of antidepressant, anxiolytic, and antipsychotic medication use in individuals with Williams syndrome: effectiveness and adverse effects. Res Dev Disabil 33: 2106-2121.

21. Bertelli MO, Rossi M, Scuticchio D, Bianco A (2015) Diagnosing psychiatric disorders in people with intellectual disabilities: issues and achievements. Advances in Mental Health and Intellectual Disabilities 9: 230-242.

Copyright: (C2019 Beghi M. This is an open-access article distributed under the terms of the Creative Commons Attribution License, which permits unrestricted use, distribution, and reproduction in any medium, provided the original author and source are credited. 